\title{
ESTIMATION OF CARBON SEQUESTRATION RATE OF URBAN PARK WITH LINEAR AND CURVILINEAR DESIGN LANDSCAPE SETTING
}

\author{
OTHMAN, R. $.^{*}-$ SUID, S. ${ }^{1}-$ MOHD NOOR, N. F. ${ }^{1}-$ BAHARUDDIN, Z. M. ${ }^{1}-$ HASHIM, K. S. H. Y. ${ }^{2}$ \\ - LUKMAN HAKIM MAHAMOD, L. H. ${ }^{3}$ \\ ${ }^{1}$ International Institute for Halal Research and Training (INHART) \\ Department of Landscape Architecture, Kulliyyah of Architecture and Environmental Design \\ (KAED), International Islamic University Malaysia, 53100 Kuala Lumpur, Malaysia \\ ${ }^{2}$ Department of Urban \& Regional Planning, Kulliyyah of Architecture and Environmental \\ Design (KAED), International Islamic University Malaysia, 53100 Kuala Lumpur, Malaysia \\ ${ }^{3}$ Department of Quantity Surveying, Kulliyyah of Architecture and Environmental Design \\ (KAED), International Islamic University Malaysia, 53100 Kuala Lumpur, Malaysia \\ Corresponding author \\ e-mail: rashidi@iium.edu.my; phone: +60-126-644-772; fax: +60-361-964-864
}

(Received 24 ${ }^{\text {th }}$ Jan 2019; accepted $3^{\text {rd }}$ May 2019)

\begin{abstract}
The need for urban parks became greater as cities expanded and the urban population rapidly grew. Therefore, having an urban park can facilitate a reduction in GHG emissions by alleviating some of the impacts of this dense development. Trees in the urban parks are an important factors reducing the amount of carbon dioxide accumulated in the urban area. The carbon sequestration rate was calculated with biomass equations, using field data collection, measurements and survey data analysis. This study aimed to calculate, predict and compare carbon sequestration rate of plant materials with linear and curvilinear design landscape setting. The decisive outcome of this study are the optimization of carbon sequestration rate by selecting the right plant material specifications with suitable landscape design setting. The findings revealed that the curvilinear design landscape setting sequesters more carbon per $\mathrm{m}^{2}$ than linear design landscape setting. Plants with bigger girth and larger quantities contribute to sequestering greater carbon compared to smaller girth and fewer trees. These findings will become a green practice approached towards building a sustainable environment with better design solutions.
\end{abstract}

Keywords: carbon stock, air pollution, green technology, phytosequestration, urban landscape design

\section{Introduction}

Urban parks have been viewed as an important component of the urban fabric that benefits for community development by providing space for recreation and leisure. From a social perspective, green spaces provide health and a range of recreational and psychological benefits, create environmental awareness and encourage positive actions toward climate change (Pataki et al., 2011; Demuzere et al., 2014). A study conducted in Helsinki, Finland, indicated that nearly all $(97 \%)$ city residents participate in some outdoor recreation during the year (Sadeghian and Vardanyan, 2013). Thus, the need for urban parks become greater as cities expanded and the urban population rapidly grew. According to Department of Statistics Malaysia (2011), in tandem with Malaysia's rapid development, the proportion of urban population increased to 71.0 percent in 2010 and this growth is expected to continue as total population increased over the years. One of the state in Malaysia with a high level of urbanization was Selangor with 91.4 percent. 
A significant increase in the urban population in a short span of time creates various problems, especially environmental problems. Therefore, under the Paris Agreement, in accordance with decisions of the United Nations Framework Convention on Climate Change (UNFCCC) (Biennial Update Report to the UNFCCC, 2015), Malaysia has committed to reducing its greenhouse gas (GHG) emissions intensity of GDP by $45 \%$ by 2030 relative to the emissions intensity of GDP in 2005. Alternative ways that have been further studied to reduce these greenhouse effects in the atmosphere is through carbon sequestration technology (Wiedman et al., 2007). Carbon sequestration is the process through which $\mathrm{CO}_{2}$ from the atmosphere is absorbed naturally through photosynthesis by the plants (Pandya et al., 2013). Trees in urban parks play an important role in the urban environment by sequestering a substantial amount of carbon dioxide from the atmosphere. Urban trees in air pollution reduction, mentioning their effects in terms of intercepting atmospheric particles and absorbing various gaseous pollutants (Yin et al., 2011). In both neighborhoods, the trees with the highest carbon stocks and sequestration rates are generally located along the main roads and in public parks (Velasco et al., 2016). Although the impacts of urban trees thus have been studied rather extensively, at least through urban quality models, there is a suggestion that research specifically on urban parks has been limited so far (Pataki et al., 2011; Yin et al., 2011). Hence, this study aimed to estimate, predict and compare carbon sequestration rate produced by plant materials through linear and curvilinear design landscape setting at two selected urban parks in Selangor. The chosen case studies for this research is differentiated between two urban parks with different landscape settings, which are linear and curvilinear design landscape setting.

\section{Materials and Method}

The first method of this study used field data collection through site inventory and analysis to estimate total carbon sequestration rate Two selected urban parks in Selangor with different landscape design settings were chosen as site studies which are Putra Heights Linear Park ( $3^{\circ} 01^{\prime} 13.1^{\prime \prime N} 101^{\circ} 34^{\prime} 31.7^{\prime \prime E}$, linear design landscape setting) and Subang Jaya Urban Forest Park $\left(3^{\circ} 03^{\prime} 19.0^{\prime \prime} \mathrm{N} 101^{\circ} 34^{\prime} 15.0^{\prime \prime} \mathrm{E}\right.$, curvilinear design landscape setting) (Fig. 1).

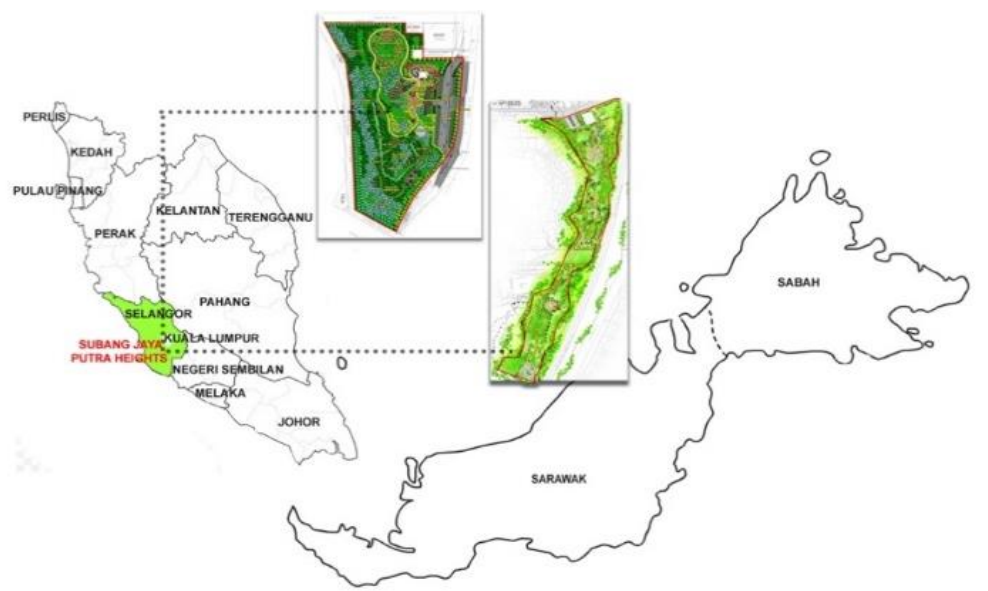

Figure 1. Base map showing Subang Jaya Urban Forest Park with curvilinear design (left) and Putra Heights Linear Park (right), Selangor, Malaysia 
Both sites were selected for inventory and analysis process in acquiring data needed. Besides, for calculating the total green area and built up area of the selected site studies, the site plan for both urban parks was obtained from the authorities. Whereas, for calculating the exact numbers of trees and detailed plants specifications on site, bill of quantities is also collected from the authorities. The data needed in this study including plants specifications such as overall plant's heights, trunk diameter, plant's age, plant's quantity and area of the plants (for climbers and turfing). Next, the current carbon sequestration rate (CSR) on every planting species was calculated using the carbon calculator formula stated in Table 1.

Table 1. The formula to calculate carbon sequestration rate (CSR)

\begin{tabular}{c|c}
\hline CSR formula for tree and shrub & CSR formula for turf, climber and groundcover \\
\hline Total Green Weight $(\mathrm{TGW}): 0.25 \mathrm{D}^{2} \mathrm{H}(1.2)$ & Total Dry Weight $(\mathrm{TDW}): 0.56 \times$ area $\left(\mathrm{m}^{2}\right)$ \\
Total Dry Weight $(\mathrm{TDW}): \mathrm{TGW} \times 0.725$ & Total Carbon Weight $(\mathrm{TCW}): \mathrm{TDW} \times 0.427$ \\
Total Carbon Weight $(\mathrm{TCW}): \mathrm{TDW} \times 0.5$ & Total $\mathrm{CO}_{2}$ Weight $\left(\mathrm{TCO}_{2} \mathrm{~W}\right): \mathrm{TCW} \times 3.6663$ \\
Total $\mathrm{CO}_{2}$ Weight $\left(\mathrm{TCO}_{2} \mathrm{~W}\right): \mathrm{TCW} \times 3.6663$ & $\mathrm{D}=$ Diameter of the trunk; $\mathrm{H}=$ Height of the tree \\
\hline
\end{tabular}

\section{Results and Discussions}

\section{Carbon sequestration rate at Putra Heights Linear Park (linear design landscape setting)}

Fig. 2 showed the planting plan at Putra Heights Linear Park which depicted linear design landscape planting. Tables 2-4 portrayed the amount of $\mathrm{CO}_{2}$ that sequestered by different types of plant species categorized under tree, shrub and turfgrass at Putra Heights Linear Park.

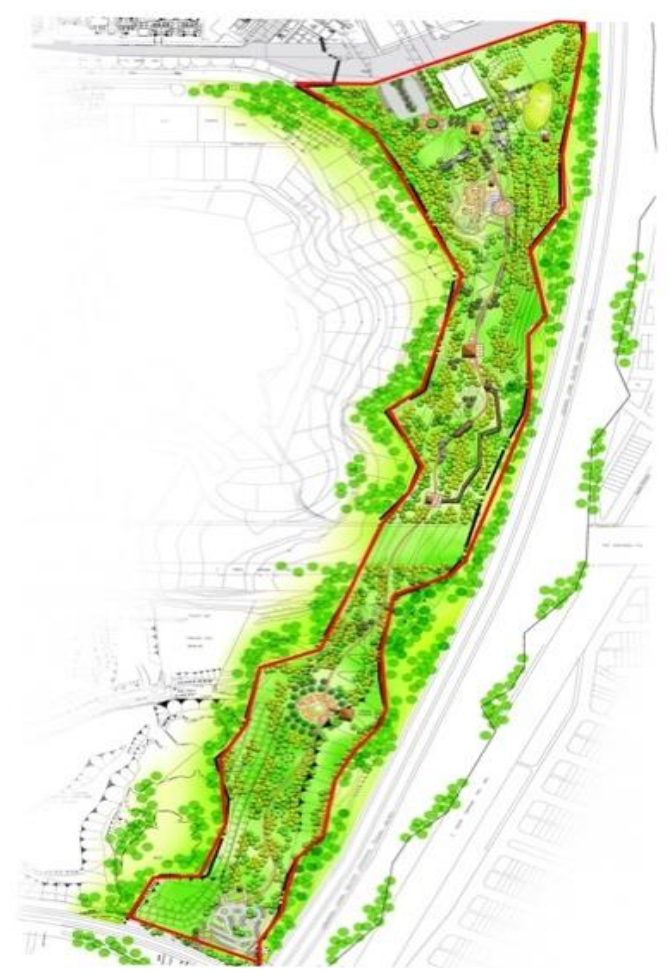

Figure 2. Planting Plan of Putra Heights Linear Park located in Selangor, Malaysia 
Table 2. Carbon sequestration rate produced by trees at Linear Park, Putra Heights

\begin{tabular}{|c|c|c|c|c|c|c|c|}
\hline NO & SPECIES & $\begin{array}{c}\text { OVERALL } \\
\text { HEIGHT } \\
\text { (feet) }\end{array}$ & $\begin{array}{c}\text { TRUNK } \\
\text { DIAMETER } \\
\text { (Inch) }\end{array}$ & $\begin{array}{l}\text { QUANTITY } \\
\text { (Nos) }\end{array}$ & AGE & $\begin{array}{c}\mathbf{t C O}_{2} \mathbf{e} \\
\text { /unit }\end{array}$ & $\begin{array}{c}\text { TOTAL } \\
\mathrm{CO}_{2} \mathrm{e}(\mathrm{kg})\end{array}$ \\
\hline 1. & Agathis borneensis & 9.84 & 1.57 & 110 & 1.6 & 0.0027 & 301.57 \\
\hline 2. & Cratoxylum cochinchinense & 13.12 & 1.97 & 55 & 2 & 0.0046 & 253.23 \\
\hline 3. & Eucalyptus deglupta & 9.84 & 1.97 & 55 & 2 & 0.0035 & 189.93 \\
\hline 4. & Eugenia grandis & 9.84 & 1.57 & 25 & 1.6 & 0.0027 & 68.54 \\
\hline 5. & Fagraea fragrans & 9.84 & 1.57 & 16 & 1.6 & 0.0027 & 43.86 \\
\hline 6. & Filicium decipiens & 9.84 & 1.77 & 50 & 1.8 & 0.0031 & 154.87 \\
\hline 7. & Gardenia carinata & 9.84 & 1.57 & 165 & 1.6 & 0.0027 & 452.36 \\
\hline 8. & Hopea odorata & 13.12 & 1.77 & 325 & 1.8 & 0.0041 & 1342.19 \\
\hline 9. & Lagerstroemia langkawiensis & 9.84 & 1.57 & 50 & 1.6 & 0.0027 & 137.08 \\
\hline 10. & Melaleuca leucadendron & 13.12 & 1.97 & 60 & 2 & 0.0046 & 276.26 \\
\hline 11. & Michelia champaca & 11.48 & 1.57 & 65 & 1.6 & 0.0032 & 207.90 \\
\hline 12. & Pongamia pinnata & 9.84 & 1.57 & 30 & 1.6 & 0.0027 & 82.25 \\
\hline 13. & Samanea saman & 22.97 & 4 & 131 & 4 & 0.0166 & 2176.79 \\
\hline 14. & Tristaniopsis whiteana & 9.84 & 1.57 & 46 & 1.6 & 0.0027 & 126.11 \\
\hline 15. & Xanthostemon chrysanthus & 9.84 & 1.57 & 25 & 1.6 & 0.0027 & 68.54 \\
\hline 16. & Cynometra cauliflora & 9.84 & 1.57 & 28 & 1.6 & 0.0027 & 76.76 \\
\hline 17. & Garcinia mangostana & 9.84 & 1.57 & 21 & 1.6 & 0.0027 & 57.57 \\
\hline 18. & Mangifera indica & 9.84 & 1.57 & 43 & 1.6 & 0.0027 & 117.89 \\
\hline 19. & Nephelium lappaceum & 9.84 & 1.57 & 37 & 1.6 & 0.0027 & 101.44 \\
\hline 20. & Phyllanthus acidus & 9.84 & 1.57 & 32 & 1.6 & 0.0027 & 87.73 \\
\hline 21. & Azadirachta exelsa & 9.84 & 1.57 & 38 & 1.6 & 0.0027 & 104.18 \\
\hline 22. & Cinnamomum iners & 9.84 & 1.57 & 35 & 1.6 & 0.0027 & 95.95 \\
\hline 23. & Cratoxylum formosum & 9.84 & 1.57 & 38 & 1.6 & 0.0027 & 104.18 \\
\hline 24. & Mesua ferrea & 9.84 & 1.57 & 53 & 1.6 & 0.0027 & 145.30 \\
\hline 25. & Acacia auriculiformis & 32.81 & 10 & 20 & 10 & 0.0356 & 712.05 \\
\hline 26. & Dyera costulata & 32.81 & 10 & 13 & 10 & 0.0356 & 462.83 \\
\hline 27. & Hevea brasiliensis & 32.81 & 10 & 42 & 10 & 0.0356 & 1495.31 \\
\hline \multirow[t]{2}{*}{28.} & Koompassia excelsa & 32.81 & 10 & 7 & 10 & 0.0356 & 249.22 \\
\hline & & & & & & & 9691.89 \\
\hline
\end{tabular}

Table 3. Carbon sequestration rate produced by shrubs at Linear Park, Putra Heights

\begin{tabular}{c|c|c|c|c|c|c|c}
\hline NO & SPECIES & $\begin{array}{c}\text { OVERALL } \\
\text { HEIGHT } \\
\text { (feet) }\end{array}$ & $\begin{array}{c}\text { TRUNK } \\
\text { DIAMETER (Inch) }\end{array}$ & $\begin{array}{c}\text { QUANTITY } \\
\text { (Nos) }\end{array}$ & AGE & $\begin{array}{c}\text { tCOe } \\
\text { /unit }\end{array}$ & $\begin{array}{c}\text { TOTAL } \\
\text { COe (kg) }\end{array}$ \\
\hline 1 & Eugenia oleana & 3.28 & 1 & 600 & 1 & 0.0006 & 355.92 \\
2 & Murraya paniculata & 1.48 & 0.5 & 5000 & 1 & 0.0001 & 334.58 \\
\hline & & & & & & & $\mathbf{6 9 0 . 4 9}$ \\
\hline
\end{tabular}

Table 4. Carbon sequestration rate produced by turfgrass at Linear Park, Putra Heights

\begin{tabular}{c|c|c|c|c}
\hline NO. & SPECIES & AREA $\left(\mathbf{M}^{\mathbf{2}}\right)$ & QUANTITY & $\begin{array}{c}\text { TOTAL } \\
\text { COe }(\mathbf{k g})\end{array}$ \\
\hline 1 & Axonopus compresus & 96300 & 96300 & 84430.00 \\
2 & Zoysia matrella & 18000 & 18000 & 15780.00 \\
\hline & & & & $\mathbf{1 0 0 , 2 1 0 . 0 0}$ \\
\hline
\end{tabular}


The highest amount of $\mathrm{CO}_{2}$ sequestered by Axonopus compressus at $100,205.17 \mathrm{kgCO}_{2} \mathrm{e}$. This amount is substantially high as compared to the other planting categories which are from tree and shrub category, sequestered at $9691.89 \mathrm{kgCO}_{2} \mathrm{e}$ and $690.49 \mathrm{kgCO}_{2} \mathrm{e}$, respectively. The main reason for this turfgrass becomes a dominant CSR agent because of the large coverage area planted with turfing at this particular site. Thus, it can be concluded that larger turfing area contributes to the greater amount of $\mathrm{CO}_{2}$ that can be sequestered at one time. The tree species that sequestered the highest total amount of carbon at this site is Samanea saman $\left(2176.79 \mathrm{kgCO}_{2} \mathrm{e}\right)$. The high CSR value of this species compared to the other tree species is due to higher plants specification used such as taller in height, bigger in trunk diameter, planted in larger quantity and had an older age (Table 2). According to Othman et al. (2016), the effect of carbon sequestration varies from the plant species, for instance, parks with trees and shrubs have the most efficient carbon sequestration effect. Meanwhile, Eugenia oleana ranked as the highest sequester agent for a shrub with the amount of $355.92 \mathrm{kgCO}_{2} \mathrm{e}$. This value is slightly higher from the other one shrub species which is Murraya paniculata with the sequestered amount of $334.58 \mathrm{kgCO}_{2} \mathrm{e}$. From Table 3, it can be clearly seen that even though the quantity of shrub for Murraya paniculata has a greater number planted on site (5000 nos), the total CSR value for this plant is still lower than Eugenia oleana which was only planted at 600 nos on site. Therefore, this finding revealed that plants specifications such as overall height and trunk diameter have very much influenced the total CSR percentage.

Fig. 3 indicates the relationship between planting categories and the total amount of carbon sequestration rate from each category. It can be depicted that the highest CSR value is from turfing category, followed by trees and shrubs category.

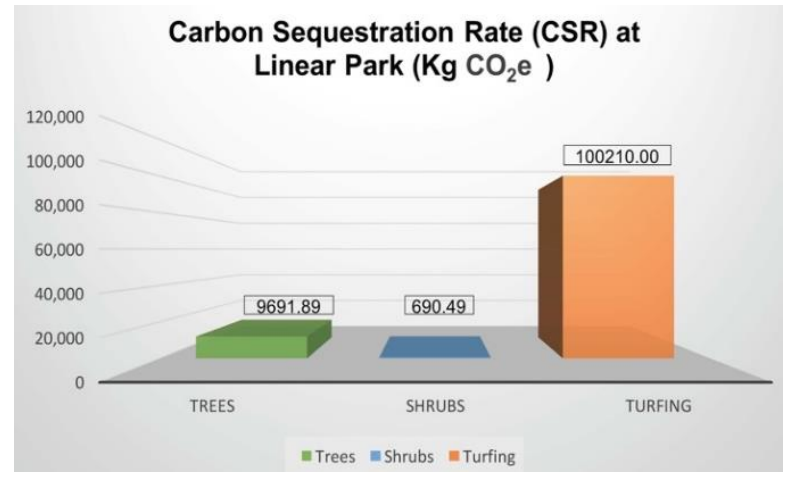

Figure 3. Carbon sequestration rate based on planting categories at Putra Heights Linear Park (linear design landscape setting)

Table 5 tabulated in detail distribution of plant's category, plant's quantity and CSR value obtained from each category. With the total numbers of 1615 nos of trees, 5600 nos of shrubs and $114,300 \mathrm{~m}^{2}$ of planted turfing, the overall total of $\mathrm{CO}_{2}$ sequestered by all plants in this site is $110,592.38 \mathrm{kgCO}_{2} \mathrm{e}$.

\section{Carbon sequestration rate at Subang Jaya urban forest park (curvilinear design landscape setting)}

Fig. 4 illustrates the planting plan at Subang Jaya urban forest park which represents curvilinear design landscape setting. Tables 6-11 showed the current carbon 
sequestration rate obtained at Subang Jaya urban forest park (curvilinear design landscape setting) based on plants categories and the total amount of $\mathrm{CO}_{2}$ sequestered by each plant. It can be concluded that the highest value represented as an effective CSR agent to sequester carbon is from turfgrass category, which named Axonopus compressus and Zoysia matrella with the sequestered amount of $24,190 \mathrm{kgCO}_{2} \mathrm{e}$ and $350 \mathrm{kgCO}_{2} \mathrm{e}$, respectively.

Table 5. Distribution of plant's quantity and carbon sequestration rate by plant's category

\begin{tabular}{c|c|c}
\hline Plant's Category & Plant's Quantity & $\begin{array}{c}\text { CSR Value/ } \\
\text { (kgCO } \mathbf{e})\end{array}$ \\
\hline Trees & $1615 \mathrm{nos}$ & $9,691.89$ \\
Shrubs & $5600 \mathrm{nos}$ & 690.49 \\
Turfing & $114300 \mathrm{~m}^{2}$ & $100,210.00$ \\
\hline Total CSR $\left(\mathrm{kgCO}_{\mathbf{2}} \mathbf{e}\right)$ & & $\mathbf{1 1 0 , 5 9 2 . 3 8}$ \\
\hline
\end{tabular}

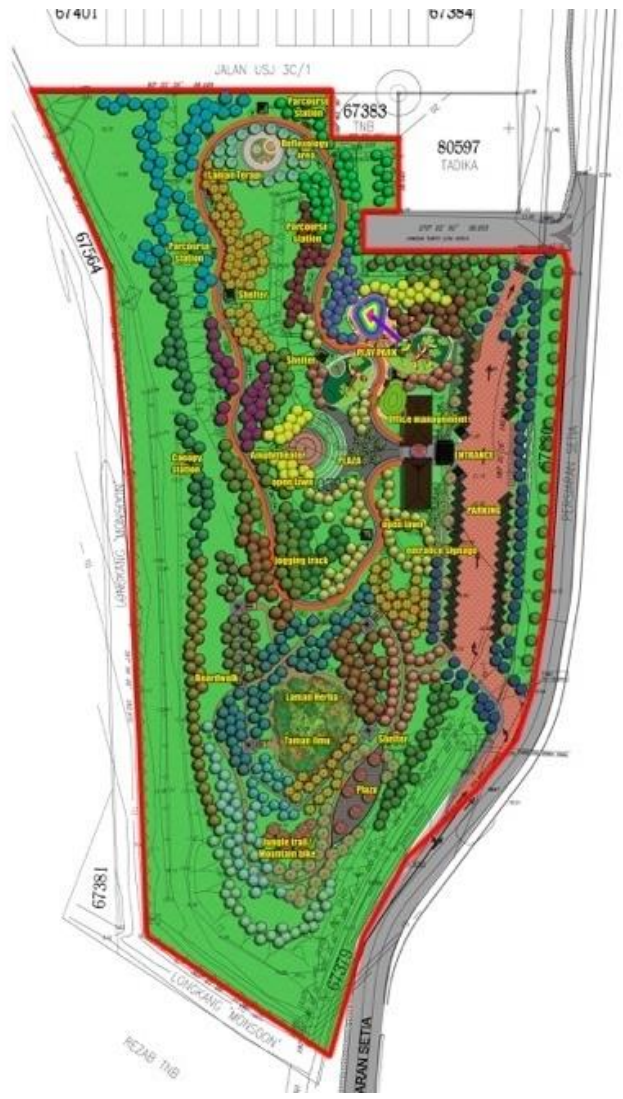

Figure 4. Planting plan of Subang Jaya urban forest park, Selangor

At this particular plant's category, Axonopus compressus has become a dominant CSR agent because of the quantity planted is tremendously high compared to Zoysia matrella. Thus, this finding proved that quantities of the plant have very much influence on the CSR value. The total amount of carbon sequestration rate produced by turfgrass at this site is $24,540 \mathrm{kgCO}_{2} \mathrm{e}$. The amount is tremendously high as compared to the other planting categories such as trees $\left(2781.28 \mathrm{kgCO}_{2} \mathrm{e}\right)$, palm $\left(170.84 \mathrm{~kg} \mathrm{CO}_{2} \mathrm{e}\right)$ and 
tall shrubs $\left(518.59 \mathrm{kgCO}_{2} \mathrm{e}\right)$ categories. Besides, for the shrubs and climbers categories have the same CSR value which is $310 \mathrm{kgCO}_{2} \mathrm{e}$ each. The tree species that sequestered the highest amount of $\mathrm{CO}_{2}$ is known as Hopea odorata $\left(274.06 \mathrm{kgCO}_{2} \mathrm{e}\right)$ whereas for the palm species is Livistonia rotundifolia $\left(170.84 \mathrm{kgCO}_{2} \mathrm{e}\right)$. Apart from that, as for the tall shrubs category, Murraya paniculata has the highest amount of $\mathrm{CO}_{2}$ sequestered. Labisia pumila $\left(90 \mathrm{kgCO}_{2} \mathrm{e}\right)$ and Tristellateia australasiae $\left(100 \mathrm{kgCO}_{2} \mathrm{e}\right)$ have ranked the highest contributor of carbon sequestration for shrubs and climbers categories.

Table 6. Carbon sequestration rate produced by trees at Subang Jaya Urban Forest Park

\begin{tabular}{|c|c|c|c|c|c|c|c|}
\hline NO & Species & $\begin{array}{l}\text { Overall Height } \\
\text { (Feet) }\end{array}$ & $\begin{array}{c}\text { Trunk } \\
\text { Diameter (Inch) }\end{array}$ & $\begin{array}{l}\text { Quantity } \\
\text { (Nos) }\end{array}$ & Age & $\mathrm{tCO}_{2} \mathrm{e} /$ unit & $\begin{array}{c}\text { Total } \\
\mathrm{CO}_{2} \mathrm{e}(\mathrm{kg})\end{array}$ \\
\hline 1. & Cinnamomum inners & 9.84 & 2 & 20 & 2 & 0.0036 & 71.18 \\
\hline 2. & Dillenia indica & 9.84 & 2 & 15 & 2 & 0.0036 & 53.39 \\
\hline 3. & Evatamia divaricata & 6.56 & 2 & 15 & 2 & 0.0024 & 35.59 \\
\hline 4. & Fragraea fragrans & 11.48 & 2 & 35 & 2 & 0.0042 & 145.33 \\
\hline 5. & Gardenia carinata & 9.84 & 2 & 30 & 2 & 0.0036 & 106.78 \\
\hline 6. & Mechelia champaka & 9.84 & 2 & 20 & 2 & 0.0036 & 71.18 \\
\hline 7. & Melia indica & 8.2 & 2 & 15 & 2 & 0.0030 & 44.49 \\
\hline 8. & Mimosup elengi & 8.2 & 2 & 20 & 2 & 0.0030 & 59.32 \\
\hline 9. & Casia fistula & 9.84 & 1.97 & 15 & 1.6 & 0.0043 & 64.75 \\
\hline 10. & Jacaranda obtusifolia & 9.84 & 1.97 & 20 & 1.97 & 0.0035 & 70.12 \\
\hline 11. & Langerstomia speciosa & 9.84 & 1.97 & 20 & 1.97 & 0.0035 & 70.12 \\
\hline 12. & Plumeriarubra & 8.2 & 2 & 15 & 2 & 0.0030 & 44.49 \\
\hline 13. & Tabeibuia rosea & 8.2 & 2 & 30 & 2 & 0.0030 & 88.98 \\
\hline 14. & Xanthostemon chrysanthus & 8.2 & 1.8 & 30 & 1.8 & 0.0027 & 80.08 \\
\hline 15. & Andira enermis & 9.84 & 1.97 & 30 & 1.97 & 0.0035 & 105.17 \\
\hline 16. & Cratoxylum cochichinensis & 11.48 & 3 & 42 & 3 & 0.0062 & 261.60 \\
\hline 17. & Dipterocarpuschartaceus & 8.2 & 2 & 25 & 2 & 0.0030 & 74.15 \\
\hline 18. & Dyeracostulata & 9.84 & 2 & 16 & 2 & 0.0036 & 56.95 \\
\hline 19. & Eucalyptus deglupta & 11.48 & 3 & 40 & 3 & 0.0062 & 249.14 \\
\hline 20. & Neobalanocarpusheimii & 9.84 & 2 & 15 & 2 & 0.0036 & 53.39 \\
\hline 21. & Hopea odorata & 11.48 & 2 & 66 & 2 & 0.0042 & 274.06 \\
\hline 22. & Melaleuca cajuputi & 9.84 & 1.97 & 25 & 1.97 & 0.0035 & 87.64 \\
\hline 23. & Mesua ferrea & 9.84 & 2 & 30 & 2 & 0.0036 & 106.78 \\
\hline 24. & Pentaspadon motley & 8.2 & 1.6 & 20 & 1.6 & 0.0024 & 47.46 \\
\hline 25. & Pometia pinnata & 9.84 & 1.97 & 20 & 1.97 & 0.0035 & 70.12 \\
\hline 26. & Shorea leprosula & 9.84 & 1.97 & 35 & 1.97 & 0.0035 & 122.70 \\
\hline 27. & Stercula foetida & 8.2 & 1.6 & 16 & 1.6 & 0.0024 & 37.96 \\
\hline 28. & Tectona grandis & 8.2 & 1.6 & 30 & 1.6 & 0.0024 & 71.18 \\
\hline 29. & Tristaniopsis whiteana & 9.84 & 2 & 40 & 2 & 0.0036 & 142.37 \\
\hline \multirow[t]{2}{*}{30.} & Eurycoma longifolia & 3.28 & 1 & 25 & 1 & 0.0006 & 14.83 \\
\hline & & & & & & & 2781.28 \\
\hline
\end{tabular}

Table 7. Carbon sequestration rate produced by palm at Subang Jaya Urban Forest Park

\begin{tabular}{c|c|c|c|c|c|c|c}
\hline NO & Species & $\begin{array}{c}\text { Overall } \\
\text { Height } \\
\text { (Feet) }\end{array}$ & $\begin{array}{c}\text { Trunk } \\
\text { Diameter } \\
\text { (Inch) }\end{array}$ & $\begin{array}{c}\text { Quantity } \\
\text { (Nos) }\end{array}$ & Age & $\begin{array}{c}\mathbf{t C O}_{\mathbf{2}} \mathbf{e} \\
/ \text { unit }\end{array}$ & $\begin{array}{c}\text { Total } \\
\mathbf{C O}_{\mathbf{2}} \mathbf{e}(\mathbf{k g})\end{array}$ \\
\hline 1. & Livistonia rotundifolia & 13.12 & 4 & 18 & 4 & 0.0095 & 170.84 \\
\hline & & & & & & & $\mathbf{1 7 0 . 8 4}$ \\
\hline
\end{tabular}


Table 8. Carbon sequestration rate produced by tall shrubs at Subang Jaya Urban Forest Park

\begin{tabular}{c|c|c|c|c|c|c|c}
\hline NO & Species & $\begin{array}{c}\text { Overall Height } \\
\text { (Feet) }\end{array}$ & $\begin{array}{c}\text { Trunk } \\
\text { Diameter (Inch) }\end{array}$ & $\begin{array}{c}\text { Quantity } \\
\text { (Nos) }\end{array}$ & Age & $\begin{array}{c}\text { tCO2e } \\
\text { /unit }\end{array}$ & $\begin{array}{c}\text { Total } \\
\text { CO2e (kg) }\end{array}$ \\
\hline 1. & Gardenia jasminoides & 1.64 & 1 & 300 & 1 & 0.00030 & 88.98 \\
2. & Jasminium sambac & 1.31 & 1 & 350 & 1 & 0.00024 & 82.92 \\
3. & Murraya paniculata & 1.64 & 1 & 350 & 1 & 0.00030 & 103.81 \\
4. & Acalypha siamensis & 1.31 & 1 & 400 & 1 & 0.00024 & 94.77 \\
5. & Bougainvillea spectabilis & 1.31 & 1 & 100 & 1 & 0.00024 & 23.69 \\
6. & Durantha erecta Gold & 1.31 & 1 & 400 & 1 & 0.00024 & 94.77 \\
7. & Leucophyllum frutescens & 1.64 & 1 & 100 & 1 & 0.00030 & 29.66 \\
\hline
\end{tabular}

Table 9. Carbon sequestration rate produced by shrubs at Subang Jaya Urban Forest Park

\begin{tabular}{c|c|c|c|c}
\hline NO. & Species & Area $\left.\mathbf{( m}^{\mathbf{2}}\right)$ & Quantity & Total CO $\mathbf{~} \mathbf{( k g )}$ \\
\hline 1. & Pandanus anaryllifolius & 33.33 & 100 & 30.00 \\
2. & Costus woodsonii & 33.33 & 100 & 30.00 \\
3. & Clinacanthu snutans & 50 & 300 & 40.00 \\
4. & Cosmos caudafusi & 50 & 300 & 40.00 \\
5. & Gynura procumbens & 50 & 300 & 40.00 \\
6. & Labisia pumila & 50 & 300 & 90.00 \\
7. & Orthosiphon aristatus & & 300 & 40.00 \\
\hline & & & $\mathbf{3 1 0 . 0 0}$ \\
\hline
\end{tabular}

Table 10. Carbon sequestration rate produced by climbers at Subang Jaya Urban Forest Park

\begin{tabular}{c|c|c|c|c}
\hline NO. & Species & Area $\left.\mathbf{( m}^{\mathbf{2}}\right)$ & Quantity & Total CO2e (kg) \\
\hline 1. & Vallaris glabra & 66.67 & 400 & 60.00 \\
2. & Portulaca grandifolia & 21.67 & 130 & 20.00 \\
3. & Thunbergia grandifolia & 50 & 300 & 40.00 \\
4. & Tristellateia australasiae & 110 & 660 & 100.00 \\
5. & Quisqualis indica & 50 & 300 & 40.00 \\
6. & Ophiopogon jaburan & 53.33 & 320 & 50.00 \\
\hline & & & $\mathbf{3 1 0 . 0 0}$ \\
\hline
\end{tabular}

Table 11. Carbon sequestration rate produced by turfgrass at Subang Jaya Urban Forest Park

\begin{tabular}{c|c|c|c|c}
\hline NO. & Species & Area $\left(\mathbf{m}^{\mathbf{2}}\right)$ & Quantity & Total CO $\mathbf{~} \mathbf{( k g )}$ \\
\hline 1. & Axonopus compressus & 27591 & 27591 & 24190.00 \\
2. & Zoysia matrella & 400 & 400 & 350.00 \\
\hline & & & & $\mathbf{2 4 5 4 0 . 0 0}$ \\
\hline
\end{tabular}

Fig. 5 showed the relationship between planting categories and the total amount of carbon sequestration rate from each category at Subang Jaya urban forest park. It can be depicted that the highest CSR value is from turfing category, followed by trees, tall shrubs, shrubs, climbers and palm category. At this particular period of time, turfing has become the dominant CSR agent due to the large coverage area for turfing. Table 12 
tabulated distribution of plant's category, quantities and the CSR value that sequestered from each planting category. With the total number of 775 nos trees, 18 nos palm, 2000 nos tall shrubs, $366.66 \mathrm{~m}^{2}$ of shrubs, $351.67 \mathrm{~m}^{2}$ of climbers and $27,991 \mathrm{~m}^{2}$ of turfing, total carbon sequestration rate for this particular site is $28,630.71 \mathrm{kgCO}_{2} \mathrm{e}$.

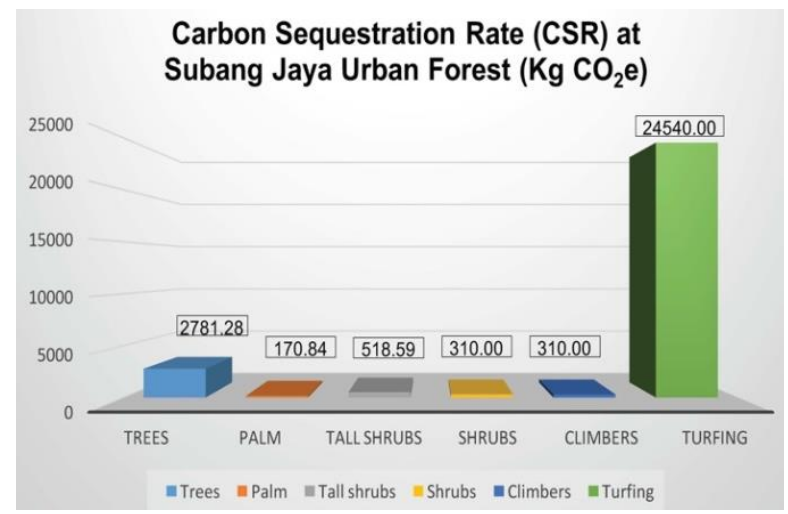

Figure 5. Carbon sequestration rate based on planting categories at Subang Jaya urban forest park (curvilinear design landscape setting)

Table 12. Distribution of plant's category, plant's quantity, andcarbon sequestration rate value

\begin{tabular}{c|c|c}
\hline Plant's Category & Plant's Quantity & CSR Value/ KgCO $\mathbf{~}_{\mathbf{2}} \mathbf{e}$ \\
\hline Trees & $775 \mathrm{nos}$ & 2781.28 \\
Palms & $18 \mathrm{nos}$ & 170.84 \\
Tall Shrubs & $2000 \mathrm{nos}$ & 518.59 \\
Shrubs & $366.66 \mathrm{~m}^{2}$ & 310.00 \\
Climbers & $351.67 \mathrm{~m}^{2}$ & 310.00 \\
Turfgrass & $27991 \mathrm{~m}^{2}$ & $24,540.00$ \\
\hline Total CSR $(\mathbf{k g C O} \mathbf{2})$ & & $\mathbf{2 8 , 6 3 0 . 7 1}$ \\
\hline
\end{tabular}

\section{Total Park Area, Green Area and Built Up Area}

Fig. 6 illustrated the total park area, green area and built up area for both selected site studies. It has been found that the green area for Putra Heights linear park $\left(114300 \mathrm{~m}^{2}\right)$ is enormously larger than Subang Jaya urban forest park $\left(25,446.63 \mathrm{~m}^{2}\right)$. From the results, it can be concluded that a larger green area contributes to the greater value of carbon sequestration rate.

Fig. 7 showed the comparison of carbon sequestration rate obtained at Putra Heights linear park (linear design landscape setting) and Subang Jaya urban forest park (curvilinear design landscape setting). From the bar graph shown, it can be clearly seen that the Putra Heights linear park $\left(110592.38 \mathrm{kgCO}_{2} \mathrm{e}\right)$ had sequestered more carbon compared to Subang Jaya urban forest park $\left(28630.71 \mathrm{kgCO}_{2} \mathrm{e}\right)$. There are many factors contribute to this difference which will be explained on the next page.

\section{Comparison of Carbon Sequestration Rate for Linear and Curvilinear Design Landscape Setting}

Table 13 portrayed comparison between Putra Heights linear park character and Subang Jaya urban forest park character. There are four categories considered in doing 
this assessment, which are a green area, landscape design setting, plant's category and quantity and also means trees specification. From the tabulated results, it is shown that Putra Heights linear park possessed higher amount in terms of green area, plant's category, and quantity and also mean trees specifications. Therefore, these factors have very much influenced the total CSR rate.

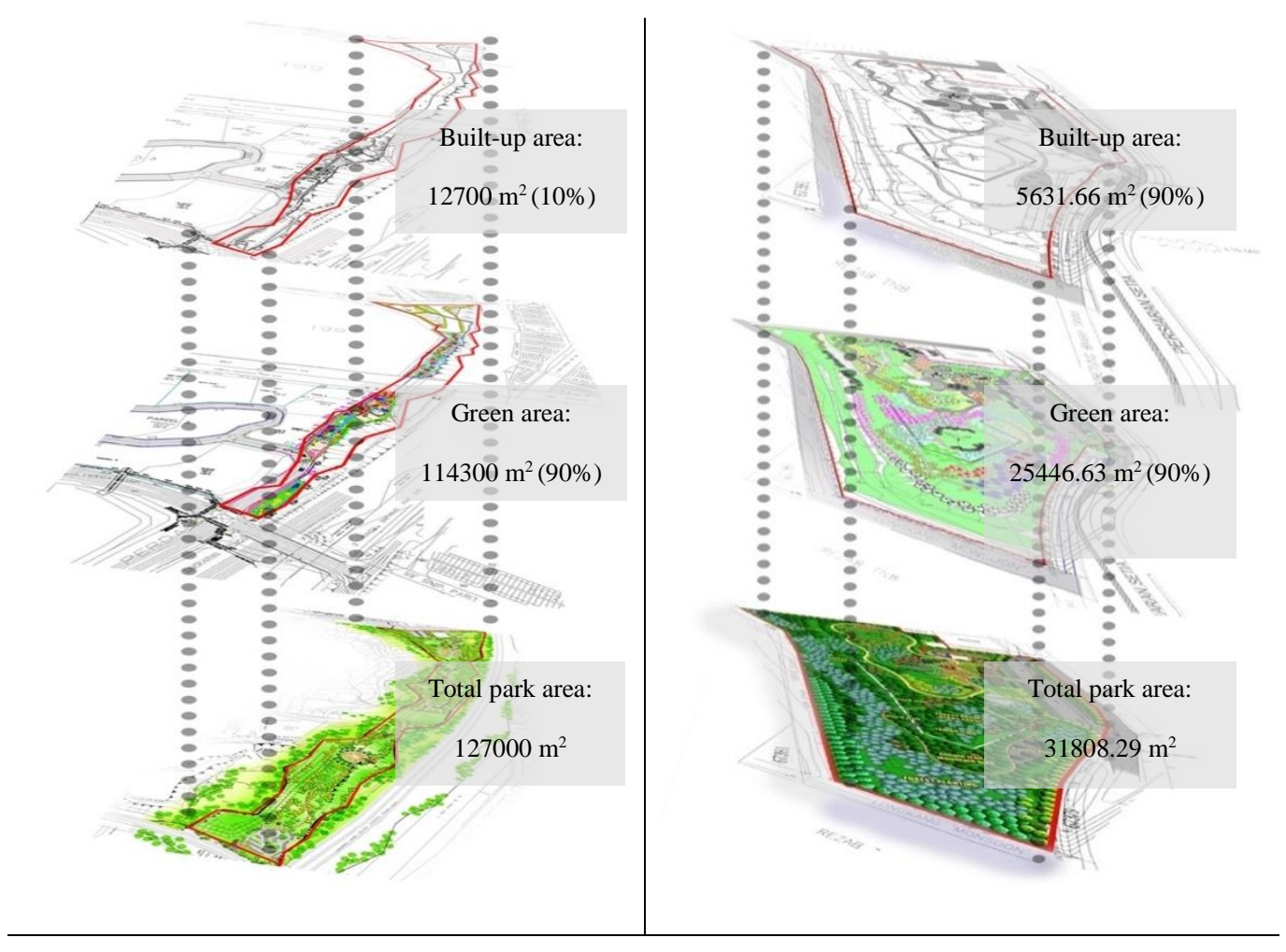

Figure 6. Distribution of total park area, green area and built up area for both urban parks

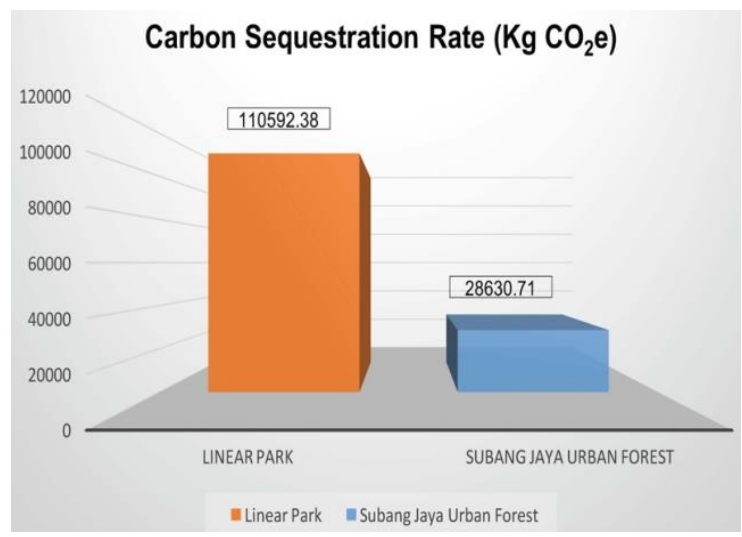

Figure 7. Carbon sequestration rate based on planting categories at Subang Jaya Urban Forest Park (curvilinear design landscape setting)

\section{Optimizing the Carbon Sequestration Rate}

According to Safaai et al. (2011), Malaysia's carbon emission is predicted at around 285.73 million tonnes by the year 2020 . With the total land area of $330,803 \mathrm{~km}^{2}$, every 
$1 \mathrm{~m}^{2}$ of Malaysia should sequester minimum of $0.863 \mathrm{kgCO}_{2} \mathrm{e}$ emissions. Next, the amount will be multiplied by the total area of the site. Table 14 showed the distribution of total CSR and $\mathrm{CO}_{2}$ sequestered per $\mathrm{m}^{2}$ for both urban parks according to planting categories. Surprisingly, Subang Jaya curvilinear urban forest park sequestered $0.9 \mathrm{kgCOe} / \mathrm{m}^{2}$, which is higher than Putra Heights linear park. Therefore, this finding revealed that curvilinear design landscape setting contributes to the greater amount of $\mathrm{CO}_{2}$ sequestered per $\mathrm{m}^{2}$. Both urban parks sequestered more than the amount of $\mathrm{CO}_{2}$ that it should sequester. Apart from that, the amount of $\mathrm{CO}_{2}$ sequestered per $\mathrm{m}^{2}$ for trees category of Subang Jaya curvilinear urban forest park $\left(0.09 \mathrm{kgCO}_{2} \mathrm{e}\right)$ is higher even though the total quantity of trees is lower compared to Putra Heights linear park $\left(0.08 \mathrm{kgCO}_{2} \mathrm{e}\right)$. There are a few factors affected such as planting distance and spatial design organization. The total amount of $\mathrm{CO}_{2}$ sequestered per $\mathrm{m}^{2}$ for turfing category is nearly equal for both parks.

Table 13. Comparison between Putra Heights linear park and Subang Jaya urban forest park landscape design setting

\begin{tabular}{c|c|c}
\hline & Putra Heights Linear Park & Subang Jaya Urban Forest Park \\
\hline Green area $\left(\mathbf{m}^{\mathbf{2}}\right)$ & $114,300.00 \mathrm{~m}^{2}$ & $25,446.63 \mathrm{~m}^{2}$ \\
\hline Landscape design setting & Linear design & Curvilinear design \\
\hline \multirow{3}{*}{ Plant's category \& quantity } & & Trees $-775 \mathrm{nos}$ \\
& Trees $-1615 \mathrm{nos}$ & Palm $-18 \mathrm{nos}$ \\
& Shrubs $-5600 \mathrm{nos}$ & Tall shrubs $-2000 \mathrm{nos}$ \\
& Turfing $-114,300 \mathrm{~m}^{2}$ & Shrubs $-366.66 \mathrm{~m}^{2}$ \\
& & Climbers $-351.67 \mathrm{~m}^{2}$ \\
Trees specification & Overall height $-14 \mathrm{ft}$ & Turfing $-24,540 \mathrm{~m}^{2}$ \\
& Trunk diameter -2.92 inch & Overall height $-9.24 \mathrm{ft}$ \\
& Age -2.9 years & Trunk diameter -1.98 inch \\
\hline
\end{tabular}

Table 14. Distribution of total CSR and $\mathrm{CO}_{2}$ Sequestered per $\mathrm{m}^{2}$ for both site studies area

\begin{tabular}{|c|c|c|}
\hline & $\begin{array}{c}\text { Total CSR } \\
\left(\mathrm{kgCO}_{2} \mathrm{e}\right)\end{array}$ & $\begin{array}{c}\mathrm{CO}_{2} \text { Sequestered per } \mathrm{m}^{2} \\
\left(\mathrm{kgCO}_{2} \mathrm{e}\right)\end{array}$ \\
\hline \multicolumn{3}{|l|}{ Putra Heights Linear Park } \\
\hline \multicolumn{3}{|c|}{ Total Park Area $=127,000 \mathrm{~m}^{2}$} \\
\hline Trees (1615 nos) & $110,592.38$ & 0.871 \\
\hline Shrubs (5600 nos) & $9,691.89$ & 0.076 \\
\hline \multirow[t]{2}{*}{ Turfing $\left(114,300 \mathrm{~m}^{2}\right)$} & 690.49 & 0.005 \\
\hline & $100,210.00$ & 0.789 \\
\hline \multicolumn{3}{|c|}{ Subang Jaya Urban Forest Park } \\
\hline \multicolumn{3}{|c|}{ Total Park Area $=31,808.29 \mathrm{~m}^{2}$} \\
\hline Trees (775 nos) & $28,630.71$ & 0.900 \\
\hline Palm (18 nos) & 2781.28 & 0.087 \\
\hline Tall Shrubs (2000 nos) & 170.84 & 0.003 \\
\hline Shrubs $\left(366.66 \mathrm{~m}^{2}\right)$ & 518.59 & 0.016 \\
\hline Climbers $\left(351.67 \mathrm{~m}^{2}\right)$ & 310.00 & 0.010 \\
\hline \multirow[t]{2}{*}{ Turfing $\left(27,991 \mathrm{~m}^{2}\right)$} & 310.00 & 0.010 \\
\hline & $24,540.00$ & \\
\hline
\end{tabular}




\section{Conclusions}

Plants in urban parks are important agents that sequester the $\mathrm{CO}_{2}$ emission of the earth atmosphere. Thus, the sequestration of carbon by urban trees and other vegetation plays an important role in mitigating climate change and have a high potential in reducing urban air pollution. Carbon sequestration rates differ based on the species of tree, planting quantity and specifications, type of plant materials group, percentage of green area and built up area as well as landscape design setting or spatial design organization. Therefore, by selecting the appropriate planting materials with suitable landscape design setting will contribute to sequestering greater value or carbon sequestration rate. It can be concluded that curvilinear design landscape setting sequester more $\mathrm{CO}_{2}$ per $\mathrm{m}^{2}$ compared to the linear design landscape setting. Thus, the selection of landscape design settings also plays an important role in contributing to the higher CSR value. Moreover, a higher percentage of the green area has much influenced in contributing to the greater CSR value. By selecting the right plant materials with higher specifications and larger quantities will also contribute to the optimum value of carbon sequestration rate in urban parks. Therefore, having an urban park with an optimum value of carbon sequestration rates will help to strengthen the ecosystem services, as a result, alleviating urban heat island and global warming. These findings will become a green practice approached towards building a sustainable environment with better design solutions. Thus, for future research, the environmental factors such as locality, type of soil and seasonal climatic variation may have influenced the carbon sequestration rate. Clearly, a further study utilizing plant material grown under different environmental conditions is required to confirm this hypothesis.

Acknowledgements. The research was supported by the Ministry of Higher Education Malaysia (MOHE) and International Islamic University Malaysia (IIUM) under research grant MOHE18-001-0001.

\section{REFERENCES}

[1] Biennial Update Report to the UNFCCC (2015): Framework Convention On Climate Change In December 2015. - Ministry Of Natural Resources and Environment Malaysia, $1-174$.

[2] Demuzere, M., Orru, K., Heidrich, O., Olazabal, E., Geneletti, D., Orru, H., Bhave, A. G., Mittal, N., Feliu, E., Faehnle, M. (2014): Mitigating and adapting to climate change: Multi-functional and multi-scale assessment of green urban infrastructure. - Journal of environmental management 146C: 107-115.

[3] Department of Statistics, Malaysia. (2011): Population Distribution and Basic Demographic Characteristic Report.

[4] Othman, R., Abu Kassim, S. Z. (2016): Assessment of plant materials carbon sequestration rate for horizontal and vertical landscape design. - International Journal of Environmental Science and Development 7(6): 410-414.

[5] Pandya Ishan, Y., Salvi, H., Chahar, O., Vaghela, N. (2013): Quantitative Analysis on Carbon Storage of 25 Valuable Tree Species of Gujrat, Incredible India. - Indian J. Sci. Res. 4(1): 137-141.

[6] Pataki, D. E., Carreiro, M. M., Cherrier, J., Grulke, N. E., Jennings, V., Pincetl, S., Pouyat, R., Whitlow, T., Zipperer, W. (2011): Coupling biogeochemical cycles in urban environments: Ecosystem services, green solutions, and misconceptions. - Frontiers in Ecology and the Environment 9(1): 27-36. 
[7] Sadeghian, M. M., Vardanyan, Z. (2013): The Benefits of Urban Parks, a Review of Urban Research. - Journal of Novel Applied Sciences: 231-237.

[8] Safaai, N. S. M., Noor, Z. Z., Hashim, H., Ujang, Z., Talib, J. (2011): Projection of $\mathrm{CO}_{2}$ emissions in Malaysia. - Environmental Progress and Sustainable Energy 30(4): 658-665.

[9] Velasco, E., Roth, M., Norford, L., Molina, L. T. (2016): Landscape and Urban Planning Does urban vegetation enhance carbon sequestration? - Landscape and Urban Planning 148: 99-107.

[10] Wiedman, T., Minx, J. (2007): The Carbon Trust Helps UK Businesses Reduce their Environmental Effect. - Ecological Economic Research Trend, Library of Congress Cataloging-In-Publication Data, Nova Science Publisher, pp.1-11.

[11] Yin, S., Shen, Z., Zhou, P., Zou, X., Che, S., Wang, W. (2011): Quantifying air pollution attenuation within urban parks: An experimental approach in Shanghai, China. Environmental Pollution 159(8-9): 2155-2163. 